\title{
ASPECTOS DA CULTURA DO CEREAL "ADLAY"
}

\author{
G. P. Viegas
}

Engenheiro agrônomo, Seçã̃o de Cereais e Leguminosas, Instituto Agronômico de Campinas

$$
1 \text { - IN'TRODUÇÃO }
$$

Devemos atribuir ao agrônomo Ubirajara Pereira Barreto (1, 2), o interêsse atualmente reinante em tôrno da cultura do cereal "adlay" (Coix lacryma-jobi L.), entre nós. O citado autor recebeu sementes da Bolívia, e talvez tenha sido influenciado pelos trabalhos do Dr. P. J. Wester, que incentivara, a partir de 1921, o cultivo dessa planta nas Filipinas. Acha que, "pelas qualidades culturais e pelo seu incomparável valor nutritivo, o "adlay" é o "primus inter pares" dos nossos cereais. E o trigo dos povos tropicais e será, indubitàvelmente, o solucionador do magno, do mais importante e mais grave problema nacional - o da nossa sadia e forte alimentação". Êssse mesmo autor dá as normas de cultivo para o cereal de porte alto, que atinge $3 \mathrm{~m}$ de altura, em Cravinhos, Estado de São Paulo, e por isso mesmo também recomendado como fonte de matéria orgânica. Nos seus trabalhos, Barreto exagera sobremodo a importância do "adlay", visto como a sua farinha não pode realmente substituir a do trigo. $O$ trigo é o único cereal cuja farinha é realmente bem panificável. A farinha de "adlay" não possui glúten, substância indispensável para a boa panificação.

Mais recentemente, Schaaffhausen $(\mathbf{9}, \mathbf{1 0})$ também se entusiasmou pelo "adlay" e tem aconselhado o plantio de uma variedade de pequeno porte, que se carateriza por produzir sementes escuras, oblongas. Ele mesmo vem utilizando o "adlay" em sua propriedade, localizada em Santo Amaro, "onde as sementes da variedade de pequeno porte tiveram sua origem", Este autor ressalta a importância dêsse cereal para o arraçoamento das aves. Baseia-se, sobretudo, neste particular, nos trabalhos que Raimo e Leme da Rocha (8), realizaram no Departamento da Produção Animal, de São Paulo. Estes últimos, tendo efetuado pesquisas a êsse respeito, concluem que o "adlay" substitui integralmente os farelos de trigo no arraçoamento das aves. Entretanto, Tôrres $\left(^{1}\right)$ aparentemente discorda dêstes autores e parece ter chegado a resultados bastante diversos.

Desconhecemos dados experimentais para ajuizar melhor da produtividade dos dois tipos de "adlay" a que nos reportamos. Ao passo que o "adlay" comum, de porte alto, a nosso ver, deve oferecer pouco interêsse

(1) Tôrres, A. Paravicini - Esc. Sup. Agric. "Luís de Queiroz", Piracicaba, em comunicação pessoal, de um trabalho no prelo. 
ao lavrador, para a produção de grãos, o de pequeno porte, também chamado "adlay" anão, nos pareceu muito mais promissor, principalmente, tendo em vista as possibilidades de colheita mecânica. Pelo que nos foi dado observar, somos de opinião que o cultivo do "adlay" é mais aconselhável para zonas de clima caraterìsticamente tropical, quente e úmido. Nas condições do planalto do Estado de São Paulo, a produção flutua muito de ano para ano, em função das condiçóes climáticas. A planta desenvolve-se e produz melhor, quando em terras de meia encosta, com suficiente umidade.

Vários outros autores se têm ocupado de incentivar o plantio do "adlay" entre nós, podendo-se mencionar, entre outros, Pestana (7), Pimentel Gomes $(4,5)$ Correia (3) e Horta (6). Este último apresenta resultados comparativos de análises do "adlay" comum com a variedade do "adlay" anão levadas a cabo no Instituto de Química Agrícola, no Rio de Janeiro.

Dentre os trabalhos publicados em outros países, devemos destacar a excelente compilação preparada por Vallayes (11), que apresenta, por seu turno, extensa bibliografia.

\section{2 - PARTE EXPERIMENTAL}

De uns anos a esta parte, resolvemos instalar alguns ensaios para estudar experimentalmente o comportamento do "adlay" de porte anão, em diversas localidades ( ${ }^{1}$ ). Apresentamos, a seguir, um resumo dos dados obtidos.

\section{1 - EPOCA DE PLANTIO}

No ano agrícola de 1948/49, foram instalados cinco ensaios, sendo um em cada uma das estações experimentais de Monte Alegre, Jaú, Capão Bonito, Mococa e Tatuí. Êsses ensaios foram plantados em quatro blocos ao acaso ; canteiros de $50 \mathrm{~m}^{2}$, isto é, com 5 linhas de $10 \mathrm{~m}$ de comprimento, espaçadas a $1 \mathrm{~m}$; colheita das 3 linhas centrais de cada canteiro.

Quadro 1.-Produção de cereal "adlay" obtida em ensaios de época de plantio instalados em diferentes localidades e em vários anos agrícolas

\begin{tabular}{|c|c|c|c|c|c|c|c|c|c|}
\hline \multirow{2}{*}{ Época de plantio } & \multicolumn{2}{|c|}{ Monte Alegre } & \multicolumn{2}{|c|}{ Tatuí } & \multicolumn{3}{|c|}{ Capão Bonito } & \multirow{2}{*}{$\frac{\text { Mococa }}{1948 / 49}$} & \multirow{2}{*}{$\frac{\text { Jaú }}{1948 / 49}$} \\
\hline & $1948 / 49$ & $1949 / 50$ & $1948 / 49$ & $1949 / 50$ & $1948 / 49$ & $1949 / 50$ & $1950 / 51$ & & \\
\hline & leg/ha & $k g / h a$ & $k g / h a$ & $\mathrm{~kg} / \mathrm{ha}$ & $k g / h a$ & $k g / h a$ & $k g / h a$ & $k g / h a$ & $k g / h a$ \\
\hline $\begin{array}{l}1 .^{\circ} \text { setembro } \\
15 \text { setembro } \\
1 .^{\circ} \text { outubro } \\
15 \text { outubro } \\
1 .^{\circ} \text { novernbro } \\
15 \text { novembro } \\
1 .^{\circ} \text { dezembro } \\
15 \text { dezembro } \\
1 .{ }^{\circ} \text { janeiro } \\
15 \text { janeiro. }\end{array}$ & $\begin{array}{r}693 \\
598 \\
959 \\
804 \\
618 \\
686 \\
779 \\
1.162 \\
702 \\
1.005\end{array}$ & $\begin{array}{l}4.570 \\
4.180 \\
4.220 \\
2.970 \\
2.250 \\
2.040 \\
1.520 \\
1.130\end{array}$ & $\begin{array}{l}2.030 \\
1.750 \\
1.630 \\
1.790 \\
1.440 \\
1.610 \\
1.530 \\
2.710 \\
1.470 \\
1.720\end{array}$ & $\begin{array}{r}2.450 \\
2.470 \\
2.870 \\
3.070 \\
2.260 \\
2.070 \\
440 \\
500\end{array}$ & $\begin{array}{l}879 \\
712 \\
917 \\
992 \\
858 \\
742 \\
742 \\
783 \\
783 \\
483\end{array}$ & $\begin{array}{r}125 \\
117 \\
92 \\
133 \\
167 \\
142 \\
125 \\
100\end{array}$ & $\begin{array}{r}-1.290 \\
1.920 \\
1.950 \\
1.160 \\
983 \\
837 \\
852 \\
\mathbf{3 8 3}\end{array}$ & $\begin{array}{r}768 \\
781 \\
1.031 \\
1.601 \\
2.012 \\
832 \\
950 \\
132 \\
607 \\
244\end{array}$ & $\begin{array}{r}2.434 \\
1.421 \\
1.033 \\
691 \\
1.623 \\
1.157 \\
900 \\
386 \\
627 \\
546\end{array}$ \\
\hline
\end{tabular}

(1) Agradecemos aos senhores chefes das estaçōes experimentais de Jaú, Capāo Bonito, Mococa e Monte Alegre, a valiosa colaborßção prestada na execução dos ensaios. 
Outros três ensaios desta natureza foram conduzidos no ano de 1949/50, adotando-se plano experimental semelhante. Em 1950/51 foi conduzido novo ensaio em Capão Bonito. Os dados obtidos figuram no quadro 1.

Analisando êstes resultados, pode-se constatar que houve grandes variações de produção entre distintas localidades e acentuadas diferenças nos diversos anos na mesma localidade. Em Capão Bonito, por exemplo, no de 1948/49 a produçã̃o de "adlay" plantado no dia 1. $917 \mathrm{~kg} / \mathrm{ha}$; no ano seguinte, foi de apenas $125 \mathrm{~kg} / \mathrm{ha}$, mas, no terceiro ano, alcançou $2.290 \mathrm{~kg} / \mathrm{ha}$. De fato, a produção do "adlay", plantado em terrenos comumente utilizados para o cultivo de milho ou algodão, está fortemente sujeita às contingências climáticas. Ele desenvolve-se e produz bem, se houver abundantes chuvas. Melhor e mais garantida será a produção, se a cultura fôr feita em solo fértil, de meia encosta com suficiente umidade. Para as condições do Estado de São Paulo, podem ser indicados os meses de outubro e novembro, como os melhores.

\section{2 - ESPAÇAMENTO E DENSIDADE DE SEMEAÇÃO}

Ainda no ano de 1948/49, foram plantados quatro ensaios para estudar o espaçamento e a densidade de semeação. Os experimentos foram conduzidos em bloco ao acaso, estudando-se os espaçamentos de 60, 80, 100 e $120 \mathrm{~cm}$ entre fileiras e quatro diferentes densidades : $8 ; 16 ; 25 ; 33$, gramas de sementes por dez metros de sulco. Os resultados obtidos figuram no quadro 2.

Quadro 2.-Produção em grãos, em quilos por hectare, dos ensaios de espaçamento e densidade de semeação de cereal "adlay", realizados em diversas localidades. em 1948/ 1949

\begin{tabular}{|c|c|c|c|c|c|}
\hline Espaçamento entre fileiras & $\begin{array}{c}\text { Densidade em } \\
10 \mathrm{~m}\end{array}$ & C. Bonito & Jaú & Mococa & Tatuí \\
\hline & & $k g / h a$ & kg/ha & $\mathrm{kg} / \mathrm{ha}$ & $k g / h a$ \\
\hline $60 \mathrm{~cm}$ - & $\begin{aligned} \mathbf{8} & -16 \\
\mathbf{1 6} & - \\
\mathbf{2 5} & - \\
\mathbf{3 3} & -\cdots\end{aligned}$ & $\begin{array}{l}1.670 \\
1.570 \\
1.570 \\
1.310\end{array}$ & $\begin{array}{r}510 \\
920 \\
1.420 \\
1.460\end{array}$ & $\begin{array}{r}1.970 \\
1.240 \\
860 \\
620\end{array}$ & $\begin{array}{l}2.220 \\
2.150 \\
2.650 \\
2.400\end{array}$ \\
\hline $80 \mathrm{~cm}-$ & 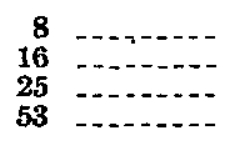 & $\begin{array}{l}1.330 \\
1.250 \\
1.200 \\
1.410\end{array}$ & $\begin{array}{r}780 \\
1.340 \\
1.340 \\
2.050\end{array}$ & $\begin{array}{r}3.040 \\
1.800 \\
1.380 \\
940\end{array}$ & $\begin{array}{l}2.290 \\
2.530 \\
2.240 \\
2.270\end{array}$ \\
\hline $100 \mathrm{~cm}$ & 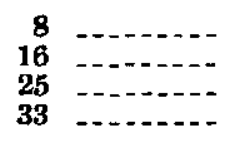 & $\begin{array}{l}1.450 \\
1.370 \\
1.400 \\
1.420\end{array}$ & $\begin{array}{l}\mathbf{6 3 0} \\
670 \\
830 \\
800\end{array}$ & $\begin{array}{l}2.490 \\
2.060 \\
1.340 \\
1.220\end{array}$ & $\begin{array}{l}2.410 \\
2.600 \\
2.740 \\
2.690\end{array}$ \\
\hline $120 \mathrm{~cm}$ & $\begin{array}{rr}8 & \\
16 & \ldots \\
25 & \cdots \\
33 & -\cdots\end{array}$ & $\begin{array}{l}1.750 \\
1.630 \\
1.710 \\
1.550\end{array}$ & $\begin{array}{r}440 \\
830 \\
710 \\
1.290\end{array}$ & $\begin{array}{l}3.270 \\
2.690 \\
1.530 \\
1.470\end{array}$ & $\begin{array}{l}1.840 \\
2.140 \\
1.850 \\
1.960\end{array}$ \\
\hline
\end{tabular}

Estudando êstes dados, pode-se afirmar que, de modo geral, foi relativamente pequena a influência do espaçamento e da densidade sôbre a pro- 
dução. Pode-se aconselhar o espaçamento de $80 \mathrm{~cm}$ entre as linhas e o plantio em filête contínuo, isto é, na densidade de $33 \mathrm{~g} / 10 \mathrm{~m}$ de sulco, ou sejam $40 \mathrm{~kg} / \mathrm{ha}$, para a variedade de porte anão. Explica êste resultado, o fato de a planta naturalmente perfilhar com maior ou menor abundância, de conformidade com o espaçamento e a densidade de plantio, dadas as naturais compensaçōes.

\section{3 - ADUBAÇÃO}

Em duas localidades - Capão Bonito e Jaú - foram conduzidos, nos últimos três anos, ensaios de adubação. O plano adotado foi o seguinte: 4 blocos ao acaso; canteiros de $50 \mathrm{~m}^{2}$ : colheita das 3 linhas centrais de cada canteiro ; adubação básica em $\mathrm{kg} / \mathrm{ha}$ : 19 de azôto ; 50 de fósforo, 25 de potássio, 400 de calcário moído. Os adubos empregados foram : resíduo de matadouro com 5 a $6 \% \mathrm{~N} ; 14-15 \% \mathrm{P}_{2} \mathrm{O}_{5}$; cloreto de potássio com $60 \%$ K.0 e calcário moído.

Os resultados obtidos em Capão Bonito e Jaú figuram no quadro 3 . Qundro 3.--Produção dos ensaios de adubação de cereal "adlay", realizados em Capão Bonito e Jaú, em 1949-51

\begin{tabular}{|c|c|c|c|c|c|c|}
\hline \multirow{2}{*}{ Tratamento } & \multicolumn{2}{|c|}{$1948 / 49$} & \multicolumn{2}{|c|}{$1949 / 50$} & \multicolumn{2}{|c|}{$1950 / 51$} \\
\hline & Capão Bonito & Jaú & Capão Bonito & Jaú & Capão Bonito & Jaú \\
\hline & $k g_{i} / h a$ & $\log / \mathrm{ha}$ & $k g / h a$ & $\mathrm{~kg} / \mathrm{ha}$ & $k g / h a$ & $k g / h a$ \\
\hline 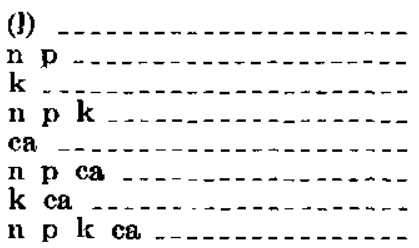 & $\begin{array}{l}2.040 \\
1.630 \\
1.720 \\
2.280 \\
2.430 \\
2.130 \\
2.200 \\
2.020\end{array}$ & $\begin{array}{l}1.510 \\
1.900 \\
1.570 \\
1.670 \\
1.330 \\
1.400 \\
1.530 \\
1.530\end{array}$ & $\begin{array}{l}517 \\
383 \\
475 \\
500 \\
442 \\
400 \\
258 \\
350\end{array}$ & $\begin{array}{l}202 \\
326 \\
229 \\
380 \\
248 \\
187 \\
333 \\
333\end{array}$ & $\begin{array}{l}1.500 \\
1.280 \\
1.330 \\
1.430 \\
1.980 \\
1.720 \\
1.380 \\
1.540\end{array}$ & $\begin{array}{l}1.730 \\
1.800 \\
1.630 \\
1.930 \\
1.920 \\
1.720 \\
1.850 \\
2.030\end{array}$ \\
\hline
\end{tabular}

Os dados obtidos nos três anos, em Capão Bonito, foram muito variáveis. As aplicações da adubação mineral completa (n p k) e de calcário sòzinho parecem ter dado os melhores resultados. São os únicos tratamentos que dão efeitos positivos no total dos três anos.

Em Jaú, o ensaio foi conduzido de acôrdo com plano semelhante, e os resultados parecem indicar maior reação à adubação com fósforo e azôto.

Como norma geral, pode-se aconselhar a adubação fosfatada na base de 50 quilos de $\mathrm{P}_{2} \mathrm{O}_{5}$ por hectare, ou mais, conforme o caso. A aplicação de azôto, potássio e calcário também poderá ser aconselhada, de conformidade com a fertilidade do solo a ser cultivado.

\section{3 - RESUMO E CONCLUSÕES}

A cultura do "adlay" (Coix lacryma-jobi L.) tem sido incentivada no país, onde recentemente foi encontrado um tipo de porte baixo, de cultivo 
mais fácil e mais promissor, ante às caraterísticas próprias dessa variedade. Essa planta exige abundante umidade e calor para se desenvolver satisfatòriamente. Nas condições do planalto do Estado de São Paulo, foram realizados alguns ensaios visando estudar a melhor época de plantio, espaçamento e adubação.

As colheitas foram muito variáveis de ano para ano e da localidade para localidade. Os dados obtidos permitem as seguintes conchusões preliminares :

a) O "adlay" deve ser plantado em outubro-novembro.

b) E relativamente pequena a influência do espaçamento entre linhas e da densidade de semeação nas linhas, dentro dos limites experimentalmente estudados, sôbre a produção, devida naturalmente às naturais compensações determinadas pelo maior ou menor número de perfilhos.

c) Em Capão Bonito, em solos do glacial, os dados dos ensaios de adubação, conduzidos durante três anos sucessivos no mesmo local, dão indicações de que a adubação mineral completa e o calcário sòzinho dão bons resultados, aumentando a produção. São os tratamentos que apresentam efeitos positivos no total de três anos.

d) Em Jaú, em terra roxa misturada, a maior reação parece ter sido observada para fósforo e azôto, em ensaio também plantado durante três anos consecutivos.

\section{SUMMARY}

A new variety of adlay (Cois: larmma-jobi $\mathrm{I}_{\text {., }}$ ) is being cultivated in the State of Sano raulo, its chel characteristics being small height, oblong black seeds, and fair yicld, espercially in low lands, with abundant rainfall and high temperature.

Some trials were condurted with this plant in four experiment stations of the Instituto Agronomico in the State of São Paulo. The yields were very variable from year to year and place to place. The following preliminary statements could be made with regard to highland conditions in this state: $a$ ) adlay should be planted in Octrober-Novemher ; b) spacing and rate of planting is not very important within the limits of $60-120 \mathrm{~cm}$ between rows and 8 to $33 \mathrm{~g}$ of seed per $10 \mathrm{~m}$ in the row, on account of the great tillering power of the plant : $c$ ) in poor and acid glacial soil of Capão Bonito, a fertilizer trial gave indication that complete mineral fertilizer and limestone applied every year gave good results, while in mixed terra roxa soil, in Jan, the great ter reaction wis to phosphorus and nitrogen.

\section{LITERA'TURA CITADA}

1. Barreto, U. P. O "adiay" na alimentação. Bol. do Min. Agric. (Rio de Janeiro) $34: 1-44.1945$.

2. Barreto, U. P. O cereal "adlay" e o problema nacional do pão. Bol. Dep. Nac. Prod. Vegetal, Min. Agric. (Río de Janeiro) 29 : 1-28. 1940.

3. Correia, E. T. Lágrima de N. Senhora. O Campo 12, 58-59. 1941.

4. Gomes, Pimentel. A cultura do "adlay". Vitória (S. Paulo) 13 : 18-19. 1948.

5. Gomes, Pimentel. A cultura do "adlav", trigo tropical. Chácara: e Quintais (S. Paulo) 70 : 485-486. 1944. 
6. Horta, P. P. Notas sóbre o cereal "adlay". A Lavoura (Rio de Janeiro) 52 : 17-22. 1948.

7. Pestana, A. C. O cereal "adlay". A Lavoura (Rio de Janeiro) 49 : 8-13. 1946.

8. Raimo, H. F. e Rocha, G. Leme. Contribuiçāo para o estudo dos substitutos dos farelos de trigo na alimentação das aves. Bol. Dept. Prod. Animal (S. Paulo) 11 : 85-95. 1950.

9. Schaaffhausen, R. V. Adlay - o cereal do futuro. Rev. dos Criadores (S. Paulo) 19 (5) : 54-56. 1948.

10. Schaaffhausen, R. V. Usos e vantagens do "adlay". Seleções Agrícolas (Rio de Janeiro) $4: 39-40.1949$.

11. Vallayes, G. Le Coix Lacryma - Jobi. Bulletin Agric. du Congo Belge 39 : 247-304. 1948. 